\title{
EFFET DE LA CONSOMMATION DE TOURTEAU D'ARACHIDE POLLUÉ PAR ASPERGILLUS FLAVUS CHEZ LE RUMINANT EN LACTATION
}

\author{
par \\ P.-M. FEHR, L. BERNAGE et V. VASSILOPOULOS \\ Laboratoire de Recherches à la Chaire de Zootechnie \\ I.N.A. 16, rue Claude-Bernard, Paris ( $V^{\mathrm{e}}$ ) \\ (avec la collaboration technique de L. Duvivier)
}

\section{Introduction}

En 1960, une maladie d'origine inconnue appelée "maladie $\mathrm{X}$ du dindon " provoqua de très lourdes pertes dans de nombreuses exploitations avicoles britanniques qui élevaient principalement des canards et des dindons. Sargeant et al. (1961 $a, b)$ montrèrent qu'elle était due à la consommation de tourteaux d'arachide en provenance du Brésil, pollués par Aspergillus flavus. Les résidus d'extraits chloroformiques de ces tourteaux, administrés à des canetons, font apparaître des symptômes identiques à ceux de la "maladie $\mathrm{X}$ du dindon» (Sargeant et al. $1961 \mathrm{c}$ ). Si ces extraits sont chromatographiés en couche mince, plusieurs taches fluorescentes à la lumière ultraviolette, peuvent être identifiées. Actuellement, on distingue essentiellement à partir d'extraits de tourteaux pollués, quatre taches fluorescentes correspondant aux aflatoxines $B_{1}, B_{2}, G_{1}, G_{2}$ dont la toxicité est bien établie (de Iongh et al. 1962, Carnaghan et al. 1963). L'aflatoxine $B_{1}$, qui est la plus toxique, est prise comme référence pour la mise en évidence et le dosage de la toxine.

L'intérêt porté à la pollution de l'arachide et de ses sousproduits prit rapidement une importance notable en alimentation animale et humaine quand il fut constaté que les tourteaux de nombreux pays tropicaux étaient couramment contaminés par Aspergillus flavus.

Parmi les espèces dont l'élevage offre un intérêt économique, les canetons et les dindonneaux se révélèrent être les plus sensibles à la présence d'aflatoxine dans leur alimentation. Des effets nocifs ont aussi été signalés dans de nombreuses autres espèces, en particulier chez les faisans, les poulets (Asplin et al, 1961), les porcs 
(Loosmore et Harding, 1961) et les veaux (Loosmore et Markson, 1961). Cependant, chez les ruminants adultes, aucun accident d'élevage n'a été rapporté à la suite de la consommation de tourteau d'arachide pollué.

L'aflatoxicose se caractérise au début par un manque d'appétit, un mauvais état général, une croissance ralentie ou même une perte de poids. Les animaux présentent rapidement des troubles hépatiques. La mort peut survenir parfois dans des délais assez courts.

Par ailleurs, Lancaster et al. (1961) et Lebreton et al. (19 $\overline{62})$ ont mis en évidence des effets à plus long terme de l'aflatoxine. Des rats consommant de faibles quantités de tourteau pollué présentent, après six mois, des hépatomes d'origine cancéreuse. Or, des cas de cancer hépatique chez l'homme sont très fréquents dans les pays qui produisent des arachides contaminées. Ainsi, on soupçonne l'aflatoxine d'être un agent favorable au développement du cancer du foie chez l'homme.

Comme dans les pays tempérés, les ressources protéiques sont suffisantes pour ne pas nécessiter l'incorporation de tourteaux dans l'alimentation humaine, le risque d'une intoxication directe par la consommation des produits végétaux pollués est écarté. Cependant, il n'est pas interdit de penser que l'homme peut être exposé à des dangers par la consommation de produits animaux, en particulier de viande, de lait et d'œufs, qui proviennent d'animaux nourris par une alimentation contenant de l'aflatoxine.

En France, les ruminants adultes, en particulier les femelles en lactation, sont les principaux consommateurs de tourteaux d'arachide. Les rations hivernales classiques, à base de foin ou d'ensilage, de racines de tubercules et de céréales, sont déficientes en matières azotées et sont très fréquemment rééquilibrées par un aliment concentré pouvant renfermer 20 à 40 p. 100 de tourteau d'arachide. Au cours de sa lactation, une bonne laitière peut ainsi consommer jusqu'à $150 \mathrm{~kg}$ de tourteau d'arachide.

Depuis 1963, notre laboratoire s'est intéressé au problème, important pour le consommateur humain, du passage éventuel de l'aflatoxine dans le lait de ruminant. Il était en effet naturel de soupçonner la mamelle de ruminant d'éliminer ce produit, puisque son rôle d'émonctoire de substances médicamenteuses et toxiques est bien connu. Nous avons, dans une première étape, essayé de mettre en évidence la présence d'aflatoxine dans le lait de vache et de chèvre consommant des tourteaux pollués. Nous avons, dans une seconde étape, cherché à préciser le degré de toxicité du lait sécrété par les animaux qui consomment une alimentation contaminée.

D'autres chercheurs : Allcroft et Lewis (1963), Allcroft et Carnaghan (1963), de Iongh et al. (1964 a), Van der Linde et al. (1964) ont abordé le même problème en utilisant exclusivement 
des vaches laitières. Leurs résultats seront confrontés avec les nôtres dans la discussion.

\section{Expérience sur vaches}

Pour mettre en évidence la présence d'aflatoxine dans le lait provenant d'animaux qui consomment du tourteau pollué par Aspergillus flavus, nous avons effectué une expérience sur deux vaches "Française, Frisonne Pie Noire», de quatre et cinq ans, au cours des troisième et quatrième mois de lactation.

\section{Protocole expérimental.}

Cet essai comprend, comme le montre le tableau I, une période expérimentale de vingt-cinq jours pendant laquelle le tourteau d'arachide pollué est distribué aux animaux. Cette période est encadrée par deux périodes témoins pré- et post-expérimentales où l'alimentation contient un tourteau d'arachide de composition semblable mais non pollué.

TABLEAU I

Plian expérimental

\begin{tabular}{c|c|c}
\hline \hline Périodes & Durée en jours & $\begin{array}{c}\text { Tourteau pollué } \\
\text { distribué en } g\end{array}$ \\
\hline Pré-expérimentale & 30 & 0 \\
\hline Expérimentale & 4 & 200 \\
& 4 & 600 \\
& 17 & 1000 \\
\hline Post-expérimentale & 10 & 0 \\
\hline \hline
\end{tabular}

Les régimes (tableau II) sont calculés suivant les besoins d'entretien et de production de chaque animal. La nature des aliments et la composition de la ration restent identiques pendant toute la durée de l'expérience. Le seul facteur qui varie dans l'alimentation des vaches est la présence ou l'absence d'aflatoxine dans le tourteau d'arachide.

Les deux vaches sont pesées régulièrement chaque semaine pendant toute la durée de l'expérience. Les refus sont contrôlés à chaque repas. La production de lait, le taux butyreux sont 
relevés à chaque traite et les matières azotées du lait ont été déterminées au moins une fois par semaine.

TABLEAU II

Composition DES RATIONS

\begin{tabular}{c|c|c|c}
\hline \hline & $\begin{array}{c}\text { Quantité ingérée } \\
\text { en moyenne } \\
(\mathrm{kg} / \mathrm{j})\end{array}$ & \multicolumn{2}{|c}{$\begin{array}{c}\text { Matière sèche ingérée } \\
(\mathrm{kg} / \mathrm{j})\end{array}$} \\
\cline { 3 - 4 } & $\begin{array}{c}\text { Aliments } \\
\text { Fache 180 }\end{array}$ & Vache 185 \\
\cline { 3 - 4 } Eoin - luzerne & 2,5 à 3 & 2,62 & 2,29 \\
Ensilage de maïs & 15 & 3,86 & 3,91 \\
Betteraves & 17 à 20 & 3,90 & 3,38 \\
Tourteau d'arachide & 1,4 à 1,9 & 1,23 & 0,75 \\
\hline \hline
\end{tabular}

Mise en évidence de l'aflatoxine.

Sur les échantillons de lait prélevés tous les quatre jours, nous avons cherché à mettre en évidence la présence d'aflatoxine en suivant la méthode du Tropical Products Institute (1962) mise au point pour la détection de la toxine dans le tourteau d'arachide. Nous avons pris la précaution de chromatographier l'extrait obtenu sur colonne d'alumine afin d'être sûr qu'aucun composant du lait n'atténue la netteté des taches fluorescentes d'aflatoxine sur les plaques en couche mince.

Comme dans cette première expérience, nous recherchions surtout à détecter l'aflatoxine dans les sécrétions mammaires, nous nous sommes bornés à juger l'intensité des taches d'une manière subjective.

\section{Résultats.}

L'analyse des performances des animaux rapportées dans le tableau III permet de conclure à l'absence d'une influence décelable de l'aflatoxine présente dans le régime tant sur la consommation d'aliments que sur les caractéristiques de production. Le poids des animaux, la quantité de lait, le taux butyreux, la quantité totale de matières grasses sécrétées quotidiennement par la mamelle, le niveau azoté du lait ne présentent aucune variation significative permettant de penser que la consommation d'aflatoxine modifie ces caractères. 
TABLEAU III

Production et composition du lait des vaches

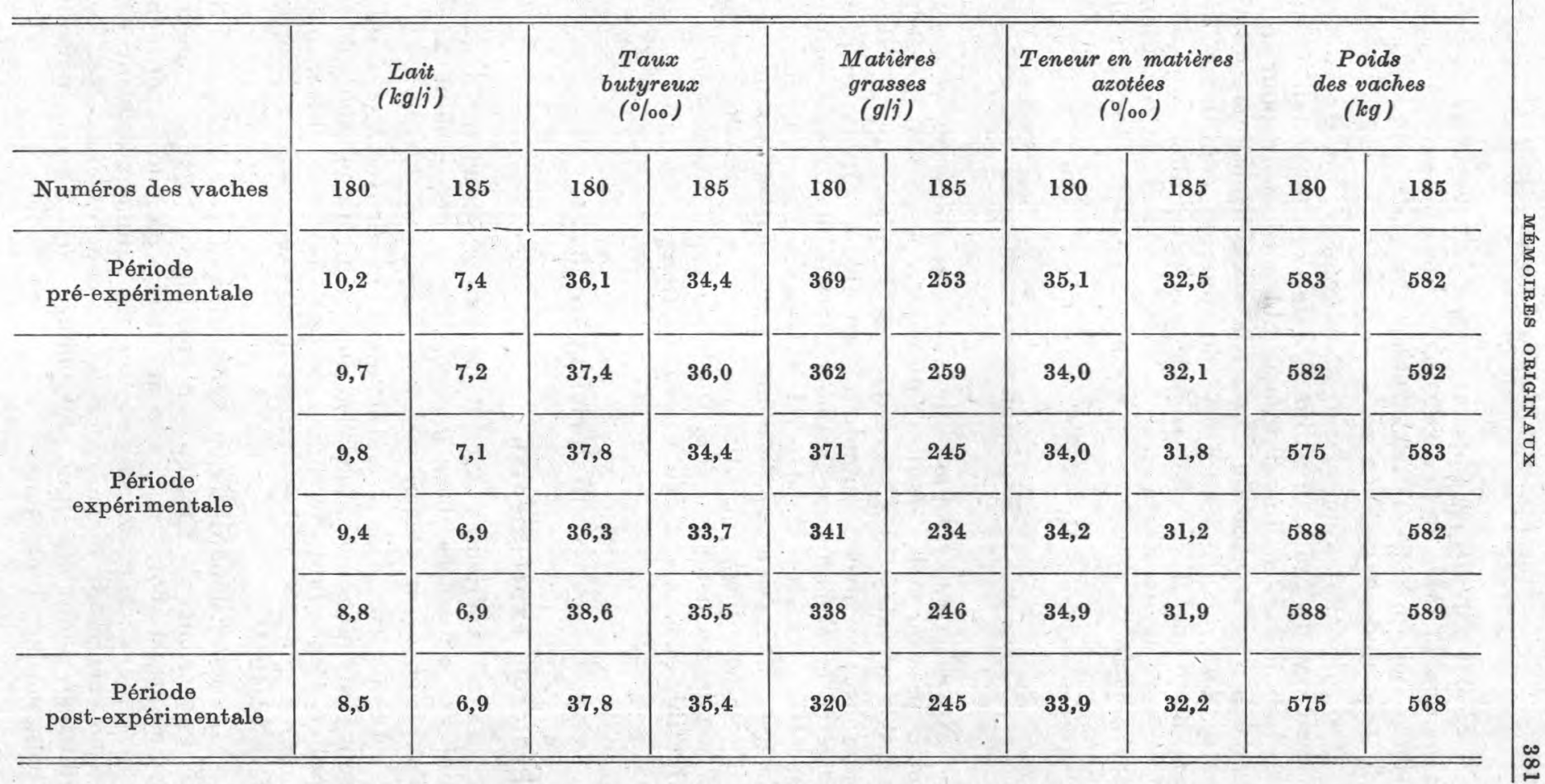


L'aflatoxine $B_{1}$ est apparue dans le lait des deux vaches comme l'indique le tableau IV. Des taches de plus faibles fluorescences sont aussi apparues à $\mathrm{R}_{\mathrm{F}} 0,2$ sur les chromato-plaques tout au long de la période expérimentale.

Ainsi, l'aflatoxine se retrouve dans le lait de vaches ayant consommé du tourteau pollué. Un délai ou une quantité minimum de toxine dans l'alimentation semble nécessaire pour qu'elle soit sécrétée par la mamelle. Quand la vache consomme quotidiennement une quantité constante de tourteau contaminé, la teneur en aflatoxine $B_{1}$ du lait semble assez variable. De plus, après l'arrêt de la distribution de la ration polluée, la sécrétion d'aflatoxine par la mamelle est encore importante pendant plusieurs jours.

\section{Expérience sur chèvres}

Nous avons entrepris deux autres expériences sur chèvres pour préciser si les résultats obtenus avaient une portée générale et s'ils n'étaient pas spécifiques de l'espèce bovine. Nous avons cherché à savoir si l'animal était sensible à la quantité d'aflatoxine ingérée et si pour une même quantité de toxine consommée, la sécrétion d'aflatoxine dépendait fortement de l'animal comme le suggère l'expérience précédente. Enfin, ces études ont permis de déterminer quelle quantité de toxine la mamelle sécrète, grâce à la mise au point d'une méthode semi-quantitative simple, reproductible et bien adaptée à nos conditions de travail.

\section{Matériel et méthodes}

\section{Protocole expérimental.}

Deux expériences $A$ et $B$, portant chacune sur deux chèvres, ont été effectuées dans des conditions ressemblant à celles de l'expérience sur vaches.

La période expérimentale de l'expérience A a duré trentesept jours pendant lesquels les chèvres ingéraient $400 \mathrm{~g}$ de tourteau pollué. Cependant, au milieu de cette période, elles n'en ont consommé que $200 \mathrm{~g}$. Les rations des chèvres sont rapportées dans le tableau V.

Les conditions de l'expérience B sont peu différentes de celles de l'expérience $A$. Les deux ehèvres ingèrent par jour une quantité constante d'aflatoxine au cours de la période expérimentale de vingt jours : le taux de pollution de l'aliment concentré de cette expérience est le même que celui du concentré $A_{2}$. Mais comme le tourteau d'arachide était plus pollué (1,5 à $2 \mathrm{ppm})$, nous en avons distribué $300 \mathrm{~g}$ par jour. 
TABLEAU IV

SÉCRÉTION D'AFLATOXINE DANS LE LAIT DE VACHE

\begin{tabular}{|c|c|c|c|c|}
\hline \multirow{2}{*}{ Périodes } & \multirow{2}{*}{$\begin{array}{c}\text { Dates de } \\
\text { prélèvement }\end{array}$} & \multirow{2}{*}{$\begin{array}{l}\text { Tourteau } \\
\text { pollué } \\
\text { distribué } \\
\text { (en } g)\end{array}$} & \multicolumn{2}{|c|}{$\begin{array}{l}\text { Apparition } \\
\text { de l'aflatoxine } B_{1} \\
\text { dans le lait }\end{array}$} \\
\hline & & & Vache 180 & Vache 185 \\
\hline $\begin{array}{l}\text { Pré-expérimentale } \\
\qquad(30 \mathrm{j})\end{array}$ & $\begin{array}{l}25^{\mathrm{e}} \text { jour } \\
29^{\mathrm{e}} \text { jour }\end{array}$ & $\begin{array}{l}0 \\
0\end{array}$ & $\begin{array}{l}0 \\
0\end{array}$ & $\begin{array}{l}0 \\
0\end{array}$ \\
\hline $\begin{array}{c}\text { Expérimentale } \\
(25 \mathrm{j})\end{array}$ & $\begin{array}{r}3^{\mathrm{e}} \text { jour } \\
7^{\mathrm{e}} \text { jour } \\
11^{\mathrm{e}} \text { jour } \\
13^{\mathrm{e}} \text { jour } \\
19^{\mathrm{e}} \text { jour } \\
23^{\mathrm{e}} \text { jour }\end{array}$ & $\begin{array}{r}200 \\
600 \\
1000 \\
1000 \\
1000 \\
1000\end{array}$ & $\begin{array}{c}0 \\
+ \\
++ \\
++ \\
T \\
+\end{array}$ & $\begin{array}{c}0 \\
++ \\
+ \\
\mathbf{T} \\
\mathbf{T} \\
+\end{array}$ \\
\hline Post-expérimentale & $\begin{array}{l}3^{e} \text { jour } \\
9^{e} \text { jour }\end{array}$ & $\begin{array}{l}0 \\
0\end{array}$ & $\begin{array}{l}++ \\
+++\end{array}$ & $\begin{array}{l}++ \\
++\end{array}$ \\
\hline $\begin{array}{ll}0 & \text { pas de fluc } \\
\mathbf{T} & \text { traces } \\
+ & \text { fluorescenc } \\
++ & \text { fluorescenc } \\
+++ & \text { fluorescenc }\end{array}$ & $\begin{array}{l}\text { escence } \\
\text { faible } \\
\text { moyenne } \\
\text { forte }\end{array}$ & & & \\
\hline
\end{tabular}

TABLEAU V

Composition des rations de L'expérience A

\begin{tabular}{|c|c|c|c|c|}
\hline Périodes & $\begin{array}{l}\text { Durée } \\
\text { (en j) }\end{array}$ & $\begin{array}{c}\text { Foin } \\
\text { (en kg) }\end{array}$ & $\begin{array}{c}\text { Pulpe } \\
\text { (en kg) }\end{array}$ & $\begin{array}{l}\text { Concentre } \\
\text { (en lkg) }\end{array}$ \\
\hline Pré-expérimentale & 30 & 1 & 0,4 & $1 \mathrm{de} T$ \\
\hline Expérimentale & $\begin{array}{r}25 \\
3 \\
10\end{array}$ & $\begin{array}{l}1 \\
1 \\
1\end{array}$ & $\begin{array}{l}0,4 \\
0,4 \\
0,4\end{array}$ & $\begin{array}{lll}1 & \text { de } & A_{2} \\
1 & \text { de } & A_{1} \\
1 & \text { de } & A_{2}\end{array}$ \\
\hline Post-expérimentale & 18 & 1 & 0,4 & $1 \mathrm{de} T$ \\
\hline
\end{tabular}

Les concentrés $T, A_{1}, A_{2}$, ont la même composition mais renferment respectivement $0,200,400 \mathrm{~g}$ de tourteau contenant 1 à $1,5 \mathrm{ppm}$ d'aflatoxine $\mathrm{B}_{1}$. 


\section{Méthode de dosage.}

La méthode de Coomes et al. (1965), mise au point pour doser l'aflatoxine $\mathrm{B}_{1}$ dans le tourteau d'arachide, est fondée sur l'extinction des taches; nous l'avons adaptée aux conditions méthodologiques que nous imposait le lait de chèvre.

A cette fin, on fait cailler $150 \mathrm{ml}$ de lait en y ajoutant $2 \mathrm{ml}$ d'acide chlorhydrique concentré. Après agitation, le lait est centrifugé pendant quinze minutes. La phase supérieure est décantée par siphonnage après avoir enlevé la couche de crème.

Le caillé est dégraissé dans un extracteur B.B.S. pendant six heures par de l'éther de pétrole. La toxine, contenue dans les résidus dégraissés et débarrassés des traces d'éther de pétrole, est extraite par du méthanol pendant quatre heures dans le même appareil.

L'extrait méthanolique est concentré jusqu'à $40 \mathrm{ml}$ environ et transvasé dans une ampoule à décanter. $30 \mathrm{ml}$ de chloroforme et $25 \mathrm{ml}$ d'eau distillée y sont ajoutés. Après agitation, la phase chloroformique est recueillie. L'extraction par $20 \mathrm{ml}$ de ehloroforme est recommencée trois fois.

Tous les extraits chloroformiques sont rassemblés et concentrés jusqu'à $0,75 \mathrm{ml}$ (solution $\mathrm{A}$ ). On dilue au dixième une fraction aliquote pour obtenir la solution B.

Des gouttes de 5 et $20 \mu \mathrm{l}$ de solution A et de solution B sont déposées sur une plaque de Kieselgel $\mathrm{K}$ préalablement conditionnée. La plaque est ensuite développée en chomatographie ascendante pendant quarante-cinq minutes environ dans un bain composé de $190 \mathrm{ml}$ de chloroforme, $10 \mathrm{ml}$ de méthanol et $2 \mathrm{ml}$ d'acide formique. Après avoir été séchée, la plaque est observée immédiatement sous lumière ultra-violette $(\lambda=365 \mathrm{~m} \mu)$.

\section{Echelle des teneurs en aflatoxine}

Tache
$20 \mu l$ sol. A
$20 \mu 1$ sol. A
$5 \mu \mathrm{sol}$. A
$20 \mu 1$ sol. B
$5 \mu \mathrm{l}$ sol. B

$$
\begin{gathered}
\text { F'luorescence } \\
\text { observée } \\
\text { à } R_{\mathrm{F}}=0,5
\end{gathered}
$$

Pas de fluorescence
Fluorescence
Fluorescenee
Fluorescence
Fluorescence

Pas de fluorescence

Tluoreseenee

Fluorescence

Fluorescence
Teneur du lait en
aflatoxine $B_{1}$

inférieure à $0,1 \mu \mathrm{g} / 1$ entre 0,4 et $0,1 \mu \mathrm{g} / 1$ entre 1 et $0,4 \mu \mathrm{g} / 1$ entre 4 et $1 \mu \mathrm{g} / 1$ supérieure à $4 \mu \mathrm{g} / 1$

Nous avons contrôlé qu'il ne restait pas d'aflatoxine dans l'extrait d'éther de pétrole et dans la solution méthanolique après le lavage au chloroforme. Cependant, il est utile de disperser correctement le caillé pour qu'il soit épuisé entièrement de son aflatoxine 
par le méthanol. Par ailleurs, aucune tache parasite, en particulier de graisses, ne perturbe la lecture de la plaque. Le passage sur colonne semble par conséquent inutile, car il n'améliore pas la détection d'aflatoxine.

Nous avons établi l'échelle des teneurs en toxine grâce à des solutions standard d'aflatoxine $B_{1}$. Nous avons vérifié que la reproductibilité des résultats obtenus par cette méthode est très satisfaisante. Elle est plus simple que les méthodes qui, comme celle de Iongh et al. (1964 b) préconisent un dégraissage après l'extraction de la toxine. En outre, le nombre limité d'opérations à effectuer diminue considérablement les risques de perte d'aflatoxine.

\section{Résultats.}

Comme nous l'avons déjà observé sur vaches, la consommation d'aflatoxine à raison de 0,4 à $0,6 \mathrm{mg}$ par jour n'a apparemment aucun effet sur la production laitière, le taux butyreux et le taux azoté du lait chez la chèvre. Dans une expérience de même nature, nous avons montré que la teneur en lactose et la composition en acides gras n'étaient pas modifiés par la présence d'aflatoxine dans le régime. Le comportement alimentaire, l'état de santé des animaux pendant toute la durée des deux expériences ont été très satisfaisants. L'estimation des refus alimentaires pendant les périodes expérimentales a montré qu'ils étaient négligeables.

Les tableaux VI et VII montrent les variations de la teneur en aflatoxine du lait respectivement au cours des expériences A et $B$.

La mamelle de chèvre comme celle de vache peut exeréter de l'aflatoxine. Après un délai de trois à cinq jours au début de la période expérimentale, le lait de chèvre renferme de l'aflatoxine $B_{1}$ déjà en proportion relativement élevée. Quand les chèvres consomment une quantité constante de toxine par jour, leur lait en renferme des teneurs fort variables. Au cours de l'expérience B, nous n'avons pu déceler la présence d'aflatoxine à la fin de la période expérimentale alors qu'au début de cette même période, une chèvre en a excrété plus de $4 \mu \mathrm{g} / 1$. Après l'arrêt de la distribution de tourteau pollué, les chèvres excrètent encore dans leur lait de l'aflatoxine pendant une période qui varie de un à dix jours.

Les deux chèvres de l'expérience $\mathrm{A}$ ont rapidement répondu à la diminution de la teneur en toxine au cours de la période expérimentale par une baisse du taux d'aflatoxine dans le lait sécrété.

Sur la plaque de chromatographie, indépendamment de la tache d'aflatoxine $B_{1}\left(R_{F} 0,5\right)$, nous avons décelé une autre tache fluorescente légèrement plus sombre à $R_{F} 0,2$. L'intensité de cette tache est toujours moins forte que celle de l'aflatoxine $B_{1}$. Elle est apparue d'une manière intermittente dans les quinze derniers jours de la période expérimentale de l'expérience A. Elle a été 
TABLEAU VI

SÉcRétion D'AFLATOXINE $B_{1}$ DANS LE LAIT DE CHłेVRE (Expérience A)

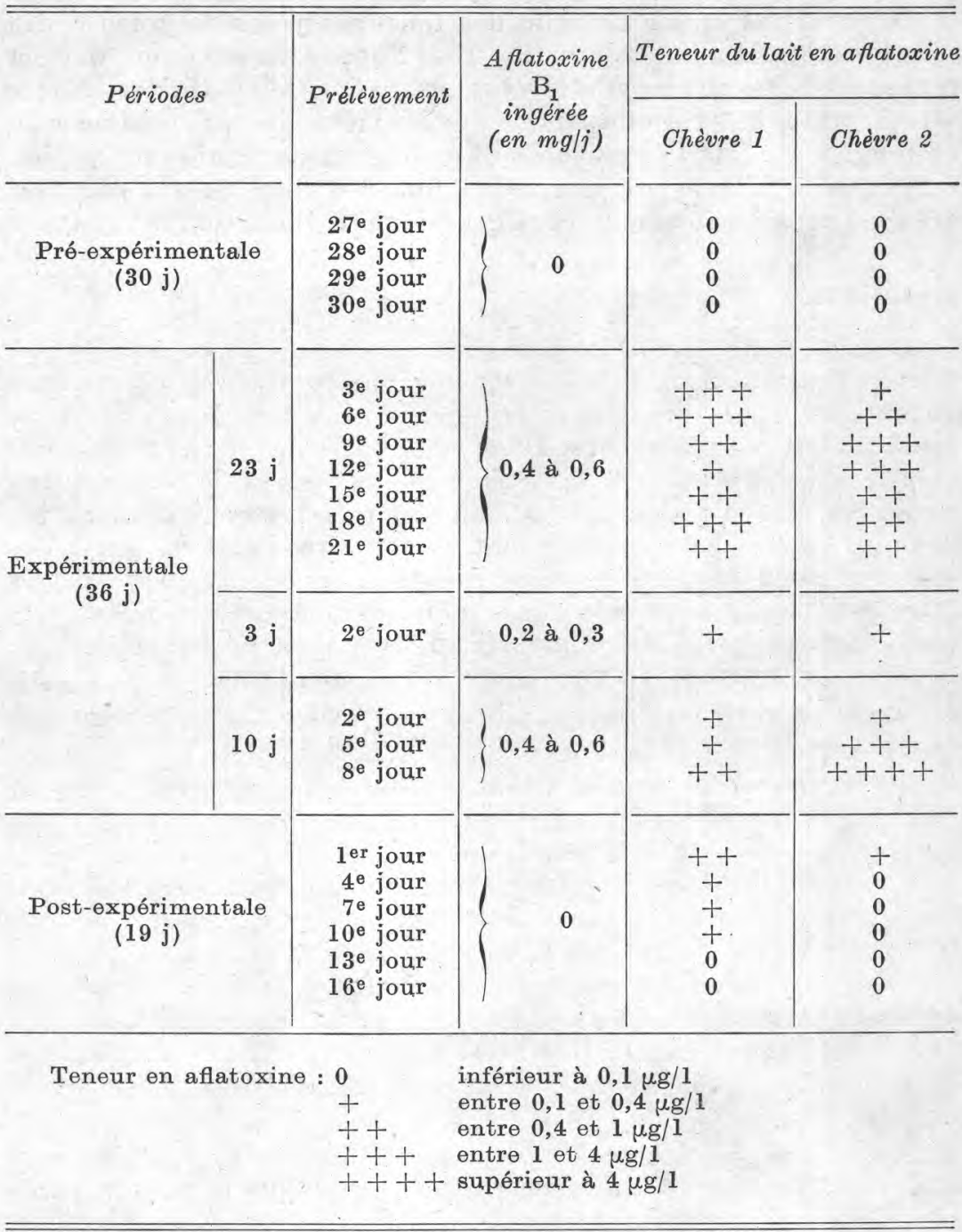

observée pendant les huit derniers jours de la période expérimentale de l'expérience $B$ avec une faible intensité.

Nous avons prélevé à plusieurs reprises des fèces et l'urine des chèvres pendant la période expérimentale. Les fèces contenaient de fortes quantités d'aflatoxine $B_{1}$ mais pas de tache à $R_{F} 0,2$. 


\section{TABLEAU VII}

SÉCRÉtion D'AFLATOXINE B DANS LE LAIT DE CHìvRE (Expérience B)

\begin{tabular}{|c|c|c|c|c|}
\hline \multirow{2}{*}{ Périodes } & \multirow{2}{*}{ Prélèvement } & \multirow{2}{*}{$\begin{array}{c}\text { A flatoxine } \\
B_{1} \\
\text { ingérée } \\
(e n m g / j)\end{array}$} & \multicolumn{2}{|c|}{ Teneur du lait en a flatoxine } \\
\hline & & & Chèvre 3 & Chèvre 4 \\
\hline $\begin{array}{l}\text { Pré-expérimentale } \\
\qquad(30 \text { j) }\end{array}$ & $\begin{array}{l}27^{e} \text { jour } \\
28^{e} \text { jour } \\
29^{\mathrm{e}} \text { jour } \\
30^{\mathrm{e}} \text { jour }\end{array}$ & 0 & $\begin{array}{l}0 \\
0 \\
0 \\
0\end{array}$ & $\begin{array}{l}0 \\
0 \\
0 \\
0\end{array}$ \\
\hline $\begin{array}{c}\text { Expérimentale } \\
(20 \mathrm{j})\end{array}$ & $\begin{array}{r}1^{\mathrm{er}} \text { jour } \\
2^{\mathrm{e}} \text { jour } \\
3^{\mathrm{e}} \text { jour } \\
4^{\mathrm{e}} \text { jour } \\
5^{\mathrm{e}} \text { jour } \\
7^{\mathrm{e}} \text { jour } \\
9^{\mathrm{e}} \text { jour } \\
11^{\mathrm{e}} \text { jour } \\
13^{\mathrm{e}} \text { jour } \\
15^{\mathrm{e}} \text { jour } \\
17^{\mathrm{e}} \text { jour } \\
19^{\mathrm{e}} \text { jour }\end{array}$ & 0,4 à 0,6 & $\begin{array}{c}0 \\
0 \\
0 \\
0 \\
++ \\
+ \\
+ \\
+ \\
+ \\
0 \\
0 \\
0\end{array}$ & $\begin{array}{c}0 \\
0 \\
0 \\
0 \\
++++ \\
++ \\
+++ \\
+++ \\
+ \\
0 \\
0 \\
0\end{array}$ \\
\hline $\begin{array}{l}\text { Post-expérimentale } \\
\qquad(10 \mathrm{j})\end{array}$ & $\begin{array}{l}1^{\text {er }} \text { jour } \\
3^{\text {e }} \text { jour } \\
7^{\text {e }} \text { jour } \\
7^{\text {e jour }} \\
9^{\text {e jour }}\end{array}$ & \} & $\begin{array}{c}++ \\
0 \\
0 \\
0 \\
0\end{array}$ & $\begin{array}{c}++ \\
+ \\
0 \\
0 \\
0\end{array}$ \\
\hline Teneur en aflatox & $\begin{aligned} e: & 0 \\
& + \\
& ++ \\
& +++ \\
& ++++\end{aligned}$ & $\begin{array}{l}\text { inférieur à } 0 \text {, } \\
\text { entre } 0,1 \text { et } \\
\text { entre } 0,4 \text { et } \\
\text { entre } 1 \text { et } 4 \\
\text { supérieur à }\end{array}$ & $\begin{array}{l}1 \mu \mathrm{g} / 1 \\
, 4 \mu \mathrm{g} / 1 \\
\mu \mathrm{g} / 1 \\
\mathrm{~g} / 1 \\
\mu \mathrm{g} / 1 .\end{array}$ & 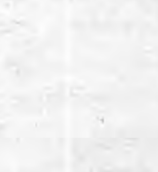 \\
\hline
\end{tabular}

Dans l'urine, nous avons aussi décelé de l'aflatoxine $\mathbf{B}_{1}$ dont la concentration est toujours nettement supérieure à celle du lait et aussi de la toxine $R_{F} 0,2$.

Les chèvres consommant par jour 0,4 à $0,6 \mathrm{mg}$ d'aflatoxine en sécrètent approximativement 0,4 à $1 \mu \mathrm{g} / \mathrm{l}$ dans le lait soit en moyenne 0,6 à $1,5 \mu \mathrm{g}$ par jour. Ainsi, le lait de ces animaux ne contient environ qu'un cinq centième de la toxine ingérée et au maximum qu'un cinquantième. 


\section{Discussion}

Dans nos conditions expérimentales, nous n'avons pas observé une influence de l'ingestion d'aflatoxine sur la consommation alimentaire, le poids et l'état général des vaches et des chèvres. La production, le taux butyreux, le taux azoté, la teneur en lactose et la composition en acides gras du lait ne sont pas perturbés.

Van der Linde et al. (1964) ont aussi observé que la production laitière des vaches n'était pas influencée par la consommation de rations qui contenaient des quantités d'aflatoxine semblables à celles de nos expériences. Par contre, Allcroft et Lewis (1963) ont constaté une chute de production de 15 p. $100 \mathrm{chez}$ les vaches qui consomment pendant une longue période une ration au moins dix fois plus polluée que la nôtre.

La relative résistance du ruminant adulte à l'aflatoxicose que nous avons observée comme Lewis et al. (1966) et l'apparente divergence entre nos résultats et ceux d'Allcroft et Lewis (1963) pourrait peut-être s'expliquer par une éventuelle action protectrice du rumen. Celle-ci deviendrait inefficace au-delà d'une certaine quantité de toxine ingérée.

Quand l'alimentation distribuée à des vaches et à des chèvres renferme du tourteau d'arachide contaminé par Aspergillus flavus, nous avons observé que le lait de ces deux espèces contient des quantités appréciables d'aflatoxine comme l'ont aussi constaté plusieurs auteurs chez les vaches (Allcroft et Lewis, 1963 ; Allcroft et Carnaghan, 1963 ; de Iongh et al. $1964 a$; Van der Linde et al., 1964). Ainsi, la mamelle de chèvre, comme celle de vache est l'un des émonctoires de l'aflatoxine.

Nous avons constaté comme de Iongh et al. (1964 a) la présence d'une substance fluorescente à la lumière ultra-violette à $R_{F}$ 0,2. Ces auteurs en ont démontré la toxicité et l'ont appelée " milktoxine " que l'on préfère désigner actuellement par aflatoxine M. Cependant, dans nos expériences, la quantité d'aflatoxine $\mathbf{B}_{\mathbf{1}}$ recueillie dans le lait est plus importante que celle qu'ils ont observée. Par contre, il semble que nous retrouvons moins d'aflatoxine M surtout chez la chèvre. En effet, chez cette espèce, nous n'avons observé de l'aflatoxine $M$ qu'après un délai variable selon les animaux, mais de l'ordre de quinze jours. Cependant, il est apparu, au cours de divers essais, que les proportions relatives en aflatoxine $B_{1}$ et en aflatoxine $\mathbf{M}$ du lait dépendaient en grande partie de l'animal.

De Iongh et al. (1964 a) ont par ailleurs mis en évidence que la ratte en lactation convertissait l'aflatoxine $B_{1}$ en aflatoxine $M$. Cette transformation se situerait dans le foie chez le rat, selon Butler et al. (1965) et chez le lapin selon Delage et Fehr (1967). Il est vraisemblable que le passage de l'aflatoxine $B_{1}$ en aflatoxine $M$ 
s'effectuerait dans les mêmes conditions chez le ruminant en lactation, néanmoins avec une plus faible intensité et un temps de latence plus important chez la chèvre. En effet, Allcroft et al (1966) en retrouvèrent dans le foie, le rein et l'urine du mouton.

Quand le taux d'aflatoxine dans la ration reste constant, sa teneur dans le lait subit d'importantes fluctuations chez le même animal. La cinétique de l'excrétion de cette toxine par la mamelle de ruminant est très différente suivant les animaux. L'animal semble pouvoir emmagasiner une partie de l'aflatoxine ingérée pour l'exeréter lorsque l'alimentation cesse d'être polluée.

Il faut noter que les quantités de toxine sécrétée par la mamelle représentent en moyenne un centième des quantités ingérées. Van der Linde et al. (1964) trouvent des teneurs dans le lait de vache sensiblement du même ordre que celles que nous avons observées chez la chèvre.

L'aflatoxine est excrétée par l'urine et par les fèces en plus forte proportion que dans le lait chez la chèvre.

Allcroft et Carnaghan (1963) ont montré les premiers, par test biologique sur canetons, que les extraits chloroformiques de lait étaient toxiques. Mais nous devons nous demander si du lait pollué, consommé en nature, est susceptible de provoquer des accidents. Le Comité Consultatif des Protéines de l'O.M.S., la F.A.O. et de l'U.N.I.C.E.F. (1966) recommande que la teneur en aflatoxine n'excède pas 0,03 à $0,05 \mathrm{ppm}$ dans les aliments servant d'apports protéiques à l'homme. La teneur en aflatoxine toujours inférieure à ces valeurs, que nous avons observée dans le lait nous incite à croire que le risque d'accidents est assez faible pour l'homme. En outre, la dilution des laits toxiques au cours des diverses opérations en laiterie tend à diminuer encore ce risque. Cependant, comme il est actuellement supposé que de faibles quantités d'aflatoxine consommées peuvent à longue échéance provoquer le cancer, il doit être recommandé d'éviter que des vaches ou des chèvres consomment du tourteau pollué par Aspergillus flavus.

\section{Résumé}

Au cours d'une expérience sur vaches et de deux expériences sur chèvres, les répercussions de la consommation de tourteau d'arachide pollué par Aspergillus flavus ont été analysées.

Une méthode d'estimation de l'aflatoxine $B_{1}$ dans le lait a été mise au point.

Aucun effet sur l'état général, la production et la composition du lait des animaux, n'a pu être décelé.

La mamelle sécrète de l'aflatoxine $B_{1}$ trois à cinq jours après le début de la distribution de tourteau pollué. L'excrétion de 
cette toxine continue après l'arrêt de la consommation du régime pollué pendant une période de trois à dix jours.

De l'aflatoxine M a été observée quinze jours environ après le début de la consommation de tourteau pollué chez la chèvre.

La cinétique de l'excrétion de l'aflatoxine par la mamelle du ruminant est très différente suivant les animaux. Quand 0,4 à $0,6 \mathrm{mg}$ de cette toxine est ingérée par une chèvre, le lait sécrété en contient de 0 à plus de $4 \mu \mathrm{g}$ par litre. En moyenne, le lait contient un centième environ de la quantité d'aflatoxine consommée.

\section{Summary}

The effect of aflatoxin-containing groundnut meal feeding has been studied in series of trials using dairy goats and cows.

A method for estimating the rate of pollution of milk with aflatoxin $B_{1}$ is described.

No effect on animals health, yield or composition of milk has been observed.

Aflatoxin $B_{1}$ appeared in milk when the animals had fed on contaminated meal for three or five days and continued to be excreted by the mammary gland when the distribution of aflatoxincontaining diet had been stopped for three or ten days.

Aflatoxin M has been observed about fifteen days after the beginning of the experiment in goat milk.

The kinetics of aflatoxin excretion by mammary gland of ruminants are very variable and dependant on animals. When 0,4 to $0,6 \mathrm{mg}$ of aflatoxin is ingested by a goat, its milk contains 0 to more $4 \mu \mathrm{g} /$ liter i.e. on an average one hundredth of the yield of fed aflatoxin.

\section{BIBLIOGRAPHIE}

Allcroft (R.) et Carnaghan (R. B. A.). Groundnut toxicity ; An examination for toxin in human food products from animals fed toxic groundnut meal. Vet. Rec., 1963, 75, 259-263.

AluCRoft (R.) et Lewis (G.), Groundnut toxicity in eattle : experimental poisoning of calves and a report on clinical effects in older cattle. Vet. Rec., 1963, 75, 487-493.

Alucroft (R.), Rogers (H.), Lewis (G.), Nabney (J.) et Best (P. E.) Metabolism of aflatoxin in sheep : excretion of the milk-toxin : Nature, 1966, 209, 154-155.

Aspin (F. D.) et CARNAGHAN (R. B. A.) The toxicity of certain groundnut meals for poultry with spécial reference to their effect on duckling and chikens. Vet. Rec., 1961, 73, 1215-1218.

BARNeS (J. M.). Report on the toxicity of aflatoxin to animals, the problem of acceptable levels of aflatoxin in food for man. Réunion du P.A.G. (W.H.O./F.A.O./U.N.I.C.E.F.), Genève, août 1966. 
BUtLer (W. H.) et Clifford (J. I.). Extraction of aflatoxin from rat liver Nature, 1965, 206, 1045-1046.

Carnaghan (R. B. A.), Hartuey (R. D.) et O'Kelity (J.).Toxicity and fluoreseence properties of the aflatoxin. Nature, 1963, 200, 1101.

Coomes (T. J.), Crowther (P. C.), Francis (B. J.) et Stevens (L.). The Detection and estimation of aflatoxin in groundnuts and groundnut materials. Part IV Routine Assessment of toxicity due to aflatoxin $\mathrm{B}_{1}$. Analyst, 1965, 90, 492-496.

Delage (J.) et Fehr (P. M.). Contribution à l'étude des répercussions de la présence d'aflatoxine dans des régimes d'animaux domestiques. Symposium sur l'aflatoxine. Villejuif, 7 mars 1966. Reproduit par Ind. Alim. Anim., 1967, no 183, 44-47.

De Iongh (H.), Berthuis (R. K.), Vles (R. C.), Barrett (C. B.) et Ord (W. O.). Investigation of the factor in groundnut meal responsible for "Turkey $\times$ disease ". Biochim. Biophys. Acta, 1962, 65, 548-550.

DE Iongh (H.), Vles (R. O.) et Van PeLt (J. G.). Milk of mammals fed an aflatoxin containing diet. Nature, $1964 a, 202,466-467$.

De Iongh (H.), Van Pelt (J. C.), Ord (W. O.) et Barrett (C. B.). A semi quantitative determination of aflatoxin $B_{1}$ in groundnut meal, groundnuts and peanut butter. Vet. Rec., 1964 b, 76, 901-903.

Lancaster (M. C.), Jenkins (F. P.), Philit (J. Me L.). Toxicity associated with certain samples of groundnut. Nature, 1961, 192, 1095.

Le Breton (E.), Fraysstnet (C.) et Boy (J.). Sur l'apparition d'hépatomes spontanés chez le rat Wistar. Rôle de la toxine de l'Aspergillus flavus. Intérêt en pathologie humaine et en cancérologie expérimentale. $C . R$. Acad. Sci. Paris, 1962, 255, 784-787.

Lewis (G.), Markson (L. M.) et Allcroft (R.). (Résultats non publiés), cités par ALLOROFT et al. 1966, Nature, 209, 154-155.

Van der Linde (J. A.), Frens (A. M.), De Iongh (H.) et Vles (R. O.). Inspection of milk from cows fed aflatoxin containing groundnut meal. Tijschr. Diergeneesk, 1964, 89, 1082-1088.

Loosmore (R. M.) et HARding (K. D. J.). A toxic factor in Brazilian groundnut causing liver damage in pigs. Vet. Rec., 1961, 73, 1362.

LOOSMORE (R. M.) et MARkson (L. M.). Poisoning of cattle by Brazilian groundnut meal. Vet. Rec., 1961, 73, 813-814.

Sargeant (K.), O'Kelit (J.), Allorott (R.) et Carnaghan (R. B. A.). A toxic factor in Brazilian groundnut meal. Vet. Rec., 1961 a, 73, 428-429.

Sargeant (K.), Sheridan (A.), O'Kelty (J.) et Carnaghan (R. B. A.). Toxicity associated with certain samples of groundnuts. Nature, $1961 b$, 192, 1096-1097.

Sargeant (K.), O'Keliy (J.), Carnaghan (R. B. A.) et Alluroft (R.). The assay of a toxie prineiple in certain groundnut meals. Vet Rec, $1961 \mathrm{c}$, 73, 1219-1223.

Tropical Products Institute Report, $n^{\circ} 25$, London, 1962. 